\title{
Lower Estimates for Certain Harmonic Functions in the Half Space
}

\author{
Gang $X u^{1}$ and Xiaoyu Zhou ${ }^{2}$ \\ ${ }^{1}$ College of Computer and Information Engineering, Henan University of Economics and Law, Zhengzhou 450000, China \\ ${ }^{2}$ College of Mathematics and Information Science, Henan University of Economics and Law, Zhengzhou 450000, China \\ Correspondence should be addressed to Gang Xu; 83041557@qq.com
}

Received 2 December 2013; Accepted 3 January 2014; Published 23 February 2014

Academic Editor: Cristina Marcelli

Copyright (c) 2014 G. Xu and X. Zhou. This is an open access article distributed under the Creative Commons Attribution License, which permits unrestricted use, distribution, and reproduction in any medium, provided the original work is properly cited.

We will give the growth properties of harmonic functions of order greater than one in a half space, which generalize the result obtained by B. Levin in a half plane.

\section{Introduction and Main Theorem}

Let $\mathbf{R}$ and $\mathbf{R}_{+}$be the sets of all real numbers and of all positive real numbers, respectively. Let $\mathbf{R}^{n}(n \geq 3)$ denote the $n$ dimensional Euclidean space with points $x=\left(x^{\prime}, x_{n}\right)$, where $x^{\prime}=\left(x_{1}, x_{2}, \ldots, x_{n-1}\right) \in \mathbf{R}^{n-1}$ and $x_{n} \in \mathbf{R}$. The boundary and closure of an open set $D$ of $\mathbf{R}^{n}$ are denoted by $\partial D$ and $\bar{D}$, respectively. The upper half space is the set $H=\left\{\left(x^{\prime}, x_{n}\right) \in\right.$ $\left.\mathbf{R}^{n}: x_{n}>0\right\}$, whose boundary is $\partial H$.

For a set $E, E \subset \mathbf{R}_{+} \cup\{0\}$, we denote $\{x \in H:|x| \in E\}$ and $\{x \in \partial H:|x| \in E\}$ by $H E$ and $\partial H E$, respectively. We identify $\mathbf{R}^{n}$ with $\mathbf{R}^{n-1} \times \mathbf{R}$ and $\mathbf{R}^{n-1}$ with $\mathbf{R}^{n-1} \times\{0\}$, writing typical points $x, y \in \mathbf{R}^{n}$ as $x=\left(x^{\prime}, x_{n}\right), y=\left(y^{\prime}, y_{n}\right)$, where $y^{\prime}=\left(y_{1}, y_{2}, \ldots, y_{n-1}\right) \in \mathbf{R}^{n-1}$, and putting

$$
\begin{gathered}
x \cdot y=\sum_{j=1}^{n} x_{j} y_{j}=x^{\prime} \cdot y^{\prime}+x_{n} y_{n}, \\
|x|=\sqrt{x \cdot x}, \quad\left|x^{\prime}\right|=\sqrt{x^{\prime} \cdot x^{\prime}}, \\
\left|x^{\prime}\right|=|x| \cos \theta, \quad x_{n}=|x| \sin \theta \quad\left(0<\theta \leq \frac{\pi}{2}\right) .
\end{gathered}
$$

Let $B_{r}$ denote the open ball with center at the origin and radius $r(>0)$ in $\mathbf{R}^{n}$. We use the standard notations $u^{+}=$ $\max (u, 0)$ and $u^{-}=-\min (u, 0)$. In the sense of Lebesgue measure $d y^{\prime}=d y_{1} \cdots d y_{n-1}$ and $d y=d y^{\prime} d y_{n}$. Let $\sigma$ denote $(n-1)$-dimensional surface area measure and let $\partial / \partial n$ denote differentiation along the inward normal into $H$.

The estimate we deal with has a long history which can be traced back to Levin's estimate of harmonic functions from below (see, e.g., [1, page 209]).

Theorem A. Let $A_{1}$ be a constant and let, $u(z)$ be harmonic in the upper half space $\mathbf{C}_{+}$and continuous on $\partial \mathbf{C}_{+}$. Suppose that

$$
\begin{gathered}
u(z) \leq A_{1} R^{\rho}, \quad z \in \mathbf{C}_{+}, R=|z|>1, \rho>1, \\
|u(z)| \leq A_{1}, \quad|z| \leq 1, \operatorname{Im} z \geq 0 .
\end{gathered}
$$

Then

$$
u\left(\operatorname{Re}^{i \varphi}\right) \geq-A_{2} A_{1}\left(1+R^{\rho}\right) \sin ^{-1} \varphi, \quad \operatorname{Re}^{i \varphi} \in \mathbf{C}_{+},
$$

where $A_{2}$ is a constant independent of $A_{1}, R, \varphi$, and the function $u(z)$.

Further versions and refinements of Theorem 1 may be found in [2, Chapter 1], [3, 4] and in the paper of KrasichkovTernovskiî [5].

In this paper, we will consider functions $u(x)$ harmonic in $H$ and continuous on $\bar{H}$. In what follows we shall denote by $M$ various values which do not depend on $K, R(=|x|), \theta$, and the function $u(x)$.

We prove in this note analogous estimates for $u(x)$ in $H$. 
Theorem 1. Suppose that

$$
\begin{gathered}
u(x) \leq K R^{\rho(R)}, \quad x \in H, \quad R=|x|>1, \quad \rho(R)>1, \\
u(x) \geq-K, \quad|x| \leq 1, \quad x_{n} \geq 0 .
\end{gathered}
$$

Then

$$
u(x) \geq-M K\left(1+\rho(R) R^{\rho(R)}\right) \sin ^{1-n} \theta,
$$

where $x \in H$ and $\rho(R)$ is nondecreasing on $[1,+\infty)$.

Remark 2. If $n=2$ and $\rho(R) \equiv \rho$, Theorem 1 is just the result of Theorem A.

Theorem 3. If (4) and (5) hold, then

$$
u(x) \geq-M K\left(1+\rho\left(\frac{N+1}{N} R\right) R^{\rho(((N+1) / N) R)}\right) \sin ^{1-n} \theta,
$$

where $x \in H, N(\geq 1)$ is a sufficiently large number, and $\rho(R)$ is defined in Theorem 1.

\section{Main Lemmas}

Carleman's formula [6] connects the modulus and the zeros of a function analytic in $\mathbf{C}_{+}$(see, e.g., [7, page 224]). Nevanlinna's formula (see [1, page 193]) refers to a harmonic function in a half disk. Ren obtained a generalized Nevanlinnatype formula in a half space and Poisson integral forumla for half balls, resepctively, which play important roles in our discussions.

Lemma 4 (see [8]). If $R>1$, then one has

$$
\begin{aligned}
& \int_{\{x \in H:|x|=R\}} u(x) \frac{n x_{n}}{R^{n+1}} d \sigma(x) \\
& \quad+\int_{\partial H(1, R)} u\left(x^{\prime}\right)\left(\frac{1}{\left|x^{\prime}\right|^{n}}-\frac{1}{R^{n}}\right) d x^{\prime}=c_{1}+\frac{c_{2}}{R^{n}},
\end{aligned}
$$

where

$$
\begin{gathered}
c_{1}=\int_{\{x \in H:|x|=1\}}\left((n-1) x_{n} u(x)+x_{n} \frac{\partial u(x)}{\partial n}\right) d \sigma(x), \\
c_{2}=\int_{\{x \in H:|x|=1\}}\left(x_{n} u(x)-x_{n} \frac{\partial u(x)}{\partial n}\right) d \sigma(x) .
\end{gathered}
$$

Lemma 5 (see [8]). Let $R>1$ and let $u(x)$ be a function in $B_{R}^{+}=B_{R} \cap H$ and continuous in $\bar{B}_{R}^{+}$. Then

$$
\begin{aligned}
u(x)= & \int_{\{y \in H:|y|=R\}} \frac{R^{2}-|x|^{2}}{\omega_{n} R} \\
& \times\left(\frac{1}{|y-x|^{n}}-\frac{1}{\left|y-x^{*}\right|^{n}}\right) u(y) d \sigma(y) \\
+ & \frac{2 x_{n}}{\omega_{n}} \int_{\partial H[0, R)}\left(\frac{1}{\left|y^{\prime}-x\right|^{n}}-\frac{R^{n}}{|x|^{n}} \frac{1}{\left|y^{\prime}-\tilde{x}\right|^{n}}\right) \\
& \times u\left(y^{\prime}\right) d y^{\prime},
\end{aligned}
$$

where $x \in B_{R}^{+}, \tilde{x}=R^{2} x /|x|^{2}, x^{*}=\left(x^{\prime},-x_{n}\right)$, and $\omega_{n}=$ $\pi^{n / 2} / \Gamma(1+(n / 2))$ is the volume of the unit $n$-ball in $\mathbf{R}^{n}$.

\section{Proof of Theorem 1}

By applying Lemma 4 to $u(x)$, we have

$$
\begin{aligned}
\int_{\{x \in H:|x|=R\}} u^{+}(x) \frac{n x_{n}}{R^{n+1}} d \sigma(x) & +\int_{\partial H(1, R)} u^{+}\left(x^{\prime}\right)\left(\frac{1}{\left|x^{\prime}\right|^{n}}-\frac{1}{R^{n}}\right) d x^{\prime} \\
= & \int_{\{x \in H:|x|=R\}} u^{-}(x) \frac{n x_{n}}{R^{n+1}} d \sigma(x) \\
& +\int_{\partial H(1, R)} u^{-}\left(x^{\prime}\right)\left(\frac{1}{\left|x^{\prime}\right|^{n}}-\frac{1}{R^{n}}\right) d x^{\prime} \\
& +c_{1}+\frac{c_{2}}{R^{n}} .
\end{aligned}
$$

It immediately follows from (4) that

$$
\begin{gathered}
\int_{\{x \in H:|x|=R\}} u^{+}(x) \frac{n x_{n}}{R^{n+1}} d \sigma(x) \leq M K R^{\rho(R)-1}, \\
\int_{\partial H(1, R)} \int u^{+}\left(x^{\prime}\right)\left(\frac{1}{\left|x^{\prime}\right|^{n}}-\frac{1}{R^{n}}\right) d x^{\prime} \leq M K R^{\rho(R)-1} .
\end{gathered}
$$

Hence from (11) and (12) we have

$$
\begin{gathered}
\int_{\{x \in H:|x|=R\}} u^{-}(x) \frac{n x_{n}}{R^{n+1}} d \sigma(x) \leq M K R^{\rho(R)-1}, \\
\int_{\partial H(1, R)} u^{-}\left(x^{\prime}\right)\left(\frac{1}{\left|x^{\prime}\right|^{n}}-\frac{1}{R^{n}}\right) d x^{\prime} \leq M K R^{\rho(R)-1} .
\end{gathered}
$$

And (14) gives

$$
\begin{aligned}
\int_{\partial H(1, R)} & \frac{u^{-}\left(x^{\prime}\right)}{\left|x^{\prime}\right|^{n}} d x^{\prime} \\
\quad \leq & \frac{2^{n}}{2^{n}-1} \int_{\partial H(1, R)} u^{-}\left(x^{\prime}\right)\left(\frac{1}{\left|x^{\prime}\right|^{n}}-\frac{1}{(2 R)^{n}}\right) d x^{\prime} \\
\quad \leq & M K \rho(R)(2 R)^{\rho(2 R)-1} .
\end{aligned}
$$
have

Since $-u(x) \leq u^{-}(x)$, by applying Lemma 5 to $-u(x)$, we

$$
-u(x) \leq I_{1}(x)+I_{2}(x),
$$


where

$$
\begin{aligned}
& I_{1}(x)=\int_{\{y \in H:|y|=R\}} \frac{R^{2}-|x|^{2}}{\omega_{n} R} \\
& \quad \times\left(\frac{1}{|y-x|^{n}}-\frac{1}{\left|y-x^{*}\right|^{n}}\right) u^{-}(y) d \sigma(y), \\
& I_{2}(x)=\frac{2 x_{n}}{\omega_{n}} \int_{\partial H[0, R)}\left(\frac{1}{\left|y^{\prime}-x\right|^{n}}-\frac{R^{n}}{|x|^{n}} \frac{1}{\left|y^{\prime}-\tilde{x}\right|^{n}}\right) \\
& \quad \times u^{-}\left(y^{\prime}\right) d y^{\prime} .
\end{aligned}
$$

We remark that

$$
\begin{gathered}
\frac{1}{|y-x|^{n}}-\frac{1}{\left|y-x^{*}\right|^{n}} \leq \frac{2 n x_{n} y_{n}}{|y-x|^{n+2}}, \\
|y-x|^{n} \geq x_{n}^{n}=|x|^{n} \sin ^{n} \theta, \quad x \in H, \quad y_{n}=0 .
\end{gathered}
$$

If we put $|x|=r>1 / 2$ and $R=2 r$ in (16), then we finally have from (13) and (18)

$$
\begin{gathered}
I_{1}(x) \leq \int_{\{y \in H:|y|=R\}} \frac{R^{2}-r^{2}}{\omega_{n} R} \frac{2 n x_{n} y_{n}}{\omega_{n}|y-x|^{n+2}} u^{-}(y) d \sigma(y) \\
\leq M K \rho(R) R^{\rho(R)} \\
I_{2}(x) \leq I_{21}(x)+I_{22}(x)
\end{gathered}
$$

where

$$
\begin{aligned}
& I_{21}(x)=\frac{2}{\omega_{n} x_{n}^{n-1}} \int_{\partial H(1, R)} u^{-}\left(y^{\prime}\right) d y^{\prime} \\
& I_{22}(x)=\frac{2}{\omega_{n} x_{n}^{n-1}} \int_{\partial H[0,1]} u^{-}\left(y^{\prime}\right) d y^{\prime}
\end{aligned}
$$

We obtain that

$$
\begin{aligned}
I_{21}(x) & \leq \frac{2 R^{n}}{\omega_{n} x_{n}^{n-1}} \int_{\partial H(1, R)} \frac{u^{-}\left(y^{\prime}\right)}{\left|y^{\prime}\right|^{n}} d y^{\prime} \\
& \leq M K \rho(R) R^{\rho(R)} \sin ^{1-n} \theta, \\
I_{22}(x) & \leq \frac{2 K}{\omega_{n} x_{n}^{n-1}} \int_{\partial H[0,1]} d y^{\prime} \\
& \leq M K \rho(R) \sin ^{1-n} \theta,
\end{aligned}
$$

from (15) and (5), respectively.

From (16), (19), and (21), we have for $|x|>1 / 2$

$$
-u(x) \leq M K \rho(R)\left(1+\rho(R) R^{\rho(R)}\right) \sin ^{1-n} \theta .
$$

For $|x| \leq 1 / 2$, we have from (5)

$$
-u(x) \leq K \leq K\left(1+\rho(R) R^{\rho(R)}\right) \sin ^{1-n} \theta .
$$

Thus the conclusion immediately follows from (22) and (23).

\section{Proof of Theorem 3}

By modifying (15), we have

$$
\begin{aligned}
& \int_{\partial H(1, R)} \frac{u^{-}\left(x^{\prime}\right)}{\left|x^{\prime}\right|^{n}} d x^{\prime} \\
& \leq \frac{(N+1)^{n}}{(N+1)^{n}-N^{n}} \int_{\partial H(1, R)} u^{-}\left(x^{\prime}\right) \\
& \quad \times\left(\frac{1}{\left|x^{\prime}\right|^{n}}-\frac{1}{(((N+1) / N) R)^{n}}\right) d x^{\prime} \\
& \leq M K \rho\left(\frac{N+1}{N} R\right)\left(\frac{N+1}{N} R\right)^{\rho(((N+1) / N) R)-1} .
\end{aligned}
$$

Then (21), (22), and (23) are replaced accordingly by the following estimates:

$$
\begin{gathered}
I_{21}(x) \leq M K \rho\left(\frac{N+1}{N} R\right)\left(\frac{N+1}{N} R\right)^{\rho(((N+1) / N) R)-1} \sin ^{1-n} \theta, \\
-u(x) \leq M K\left(1+\rho\left(\frac{N+1}{N} R\right) R^{\rho(((N+1) / N) R)}\right) \sin ^{1-n} \theta, \\
-u(x) \leq K \leq M K\left(1+\rho\left(\frac{N+1}{N} R\right) R^{\rho(((N+1) / N) R)}\right) \sin ^{1-n} \theta .
\end{gathered}
$$

All (16), (19), (25), and (21) give

$$
u(x) \geq-M K\left(1+\rho\left(\frac{N+1}{N} R\right) R^{\rho(((N+1) / N) R)}\right) \sin ^{1-n} \theta,
$$

from which the conclusion immediately follows.

\section{Conflict of Interests}

The authors declare that there is no conflict of interests regarding the publication of this paper.

\section{Acknowledgments}

The authors wish to express their appreciation to Dr. Xuna Miao for some very useful conversations related to this problem. This work was supported by the National Natural Science Foundation of China under Grants nos. 11301140 and U1304102.

\section{References}

[1] B. Ya. Levin, Lectures on Entire Functions, vol. 150, American Mathematical Society, Providence, RI, USA, 1996.

[2] N. K. Nikol'skiŭ, Selected Problems of Weighted Approximation and Spectral Analysis, vol. 120 of English Translation Proceedings of the Steklov Institute of Mathematics 1974, American Mathematical Society, Providence, RI, USA, 1976.

[3] B. Su, "Dirichlet problem for the Schrödinger operator in a half space," Abstract and Applied Analysis, vol. 2012, Article ID 578197, 14 pages, 2012. 
[4] B. Y. Su, "Growth properties of harmonic functions in the upper half-space," Acta Mathematica Sinica, vol. 55, no. 6, pp. 10951100, 2012 (Chinese).

[5] I. F. Krasichkov-Ternovski1, "Estimates for the subharmonic difference of sub-harmonic functions. II," Mathematics of the USSR-Sbornik, vol. 32, no. 1, pp. 32-59, 1977.

[6] T. Carleman, "Über die approximation analytischer funktionen durch lineare aggregate von vorgegebenen potenzen," Arkiv för Matematik, Astronomi Och Fysik, vol. 17, pp. 1-30, 1923.

[7] B. Levin, Distribution of Zeros of Entire Functions, vol. 5 of Translations of Mathematical Monographs, American Mathematical Society, Providence, RI, USA, Revised edition, 1980.

[8] Y. D. Ren, "Solving integral representations problems for the stationary Schrödinger Equation," Abstract and Applied Analysis, vol. 2013, Article ID 715252, 5 pages, 2013. 


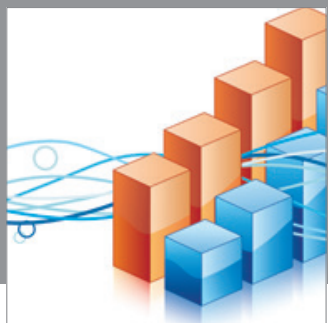

Advances in

Operations Research

mansans

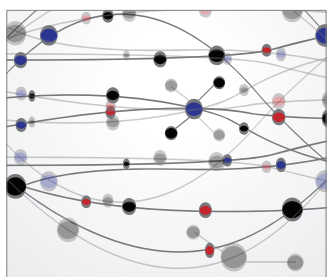

The Scientific World Journal
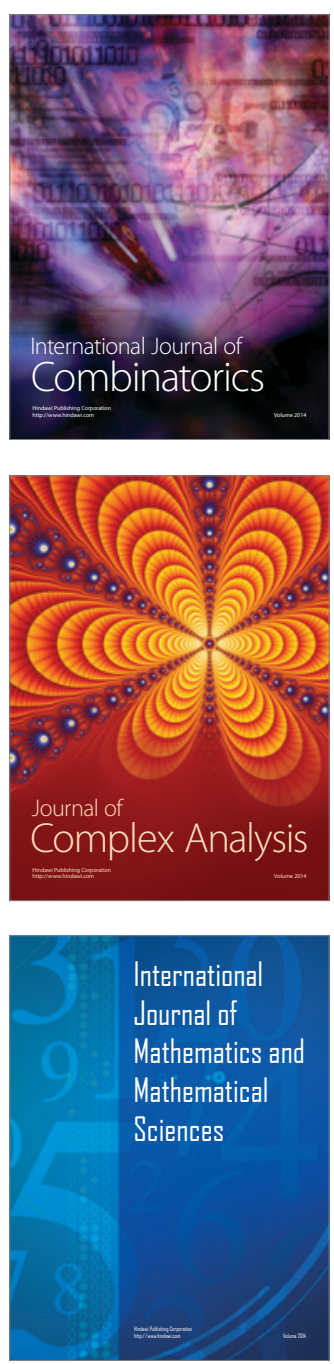
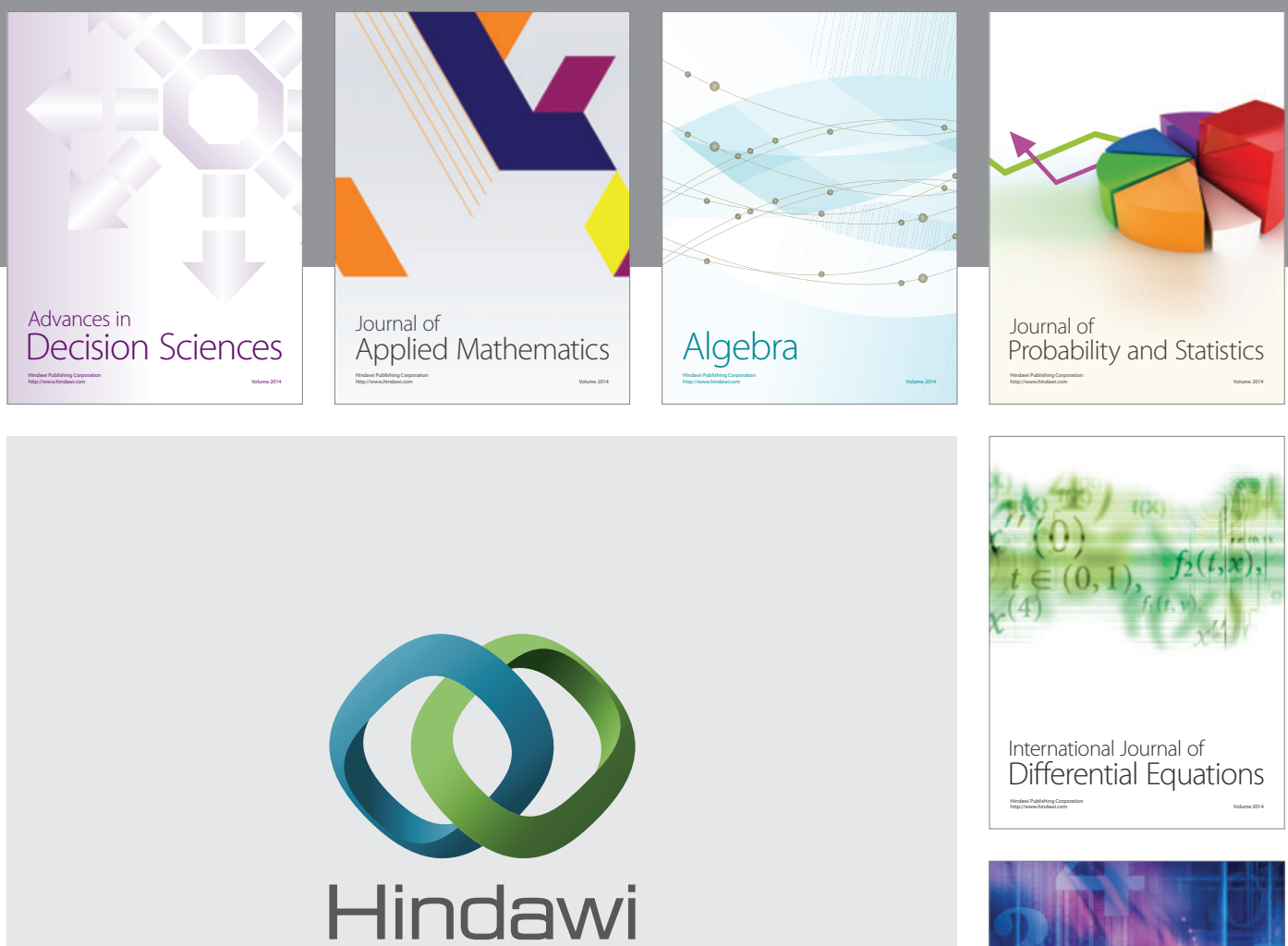

Submit your manuscripts at http://www.hindawi.com
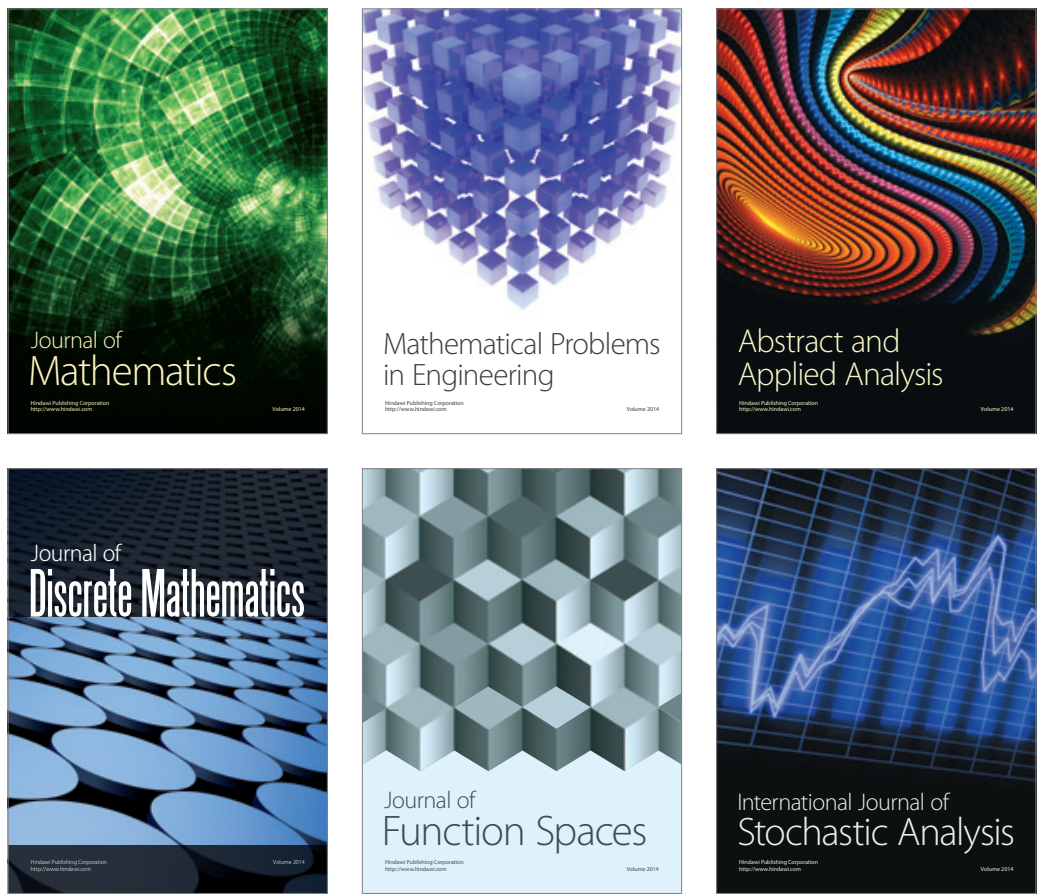

Journal of

Function Spaces

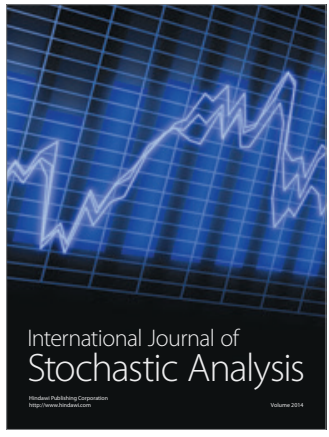

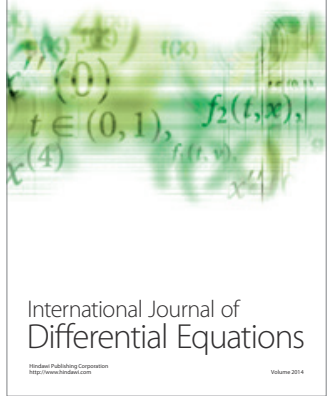
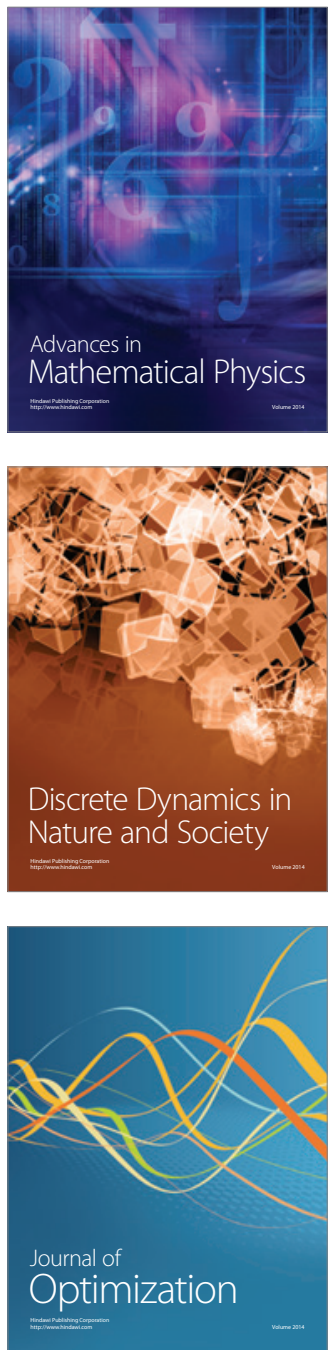